\title{
Georg Schmorl Prize of the German Spine Society (DWG) 2020: new biomechanical in vitro test method to determine subsidence risk of vertebral body replacements
}

\author{
Laura Zengerle $^{1}$ [D Christoph Fleege ${ }^{2} \cdot$ Theodor Di Pauli von Treuheim $^{1}$ (D) Daniel Vogele ${ }^{3} \cdot$ Michael Rauschmann $^{4}$. \\ Hans-Joachim Wilke ${ }^{1}$ (i)
}

Received: 21 December 2020 / Revised: 15 January 2021 / Accepted: 2 February 2021 / Published online: 17 March 2021

(c) The Author(s) 2021

\begin{abstract}
Purpose Prevention of implant subsidence in osteoporotic (thoraco)lumbar spines is still a major challenge in spinal surgery. In this study, a new biomechanical in vitro test method was developed to simulate patient activities in order to determine the subsidence risk of vertebral body replacements during physiologic loading conditions.

Methods The study included 12 (thoraco)lumbar (T11-L1, L2-L4) human specimens. After dorsal stabilisation and corpectomy, vertebral body replacements (VBR) with (a) round centrally located and (b) lateral end pieces with apophyseal support were implanted, equally distributed regarding segment, sex, mean BMD ((a) $64.2 \mathrm{mgCaHA} / \mathrm{cm}^{3}$, (b) $66.7 \mathrm{mgCaHA} /$ $\mathrm{cm}^{3}$ ) and age ((a) 78 years, (b) 73.5 years). The specimens were then subjected to everyday activities (climbing stairs, tying shoes, lifting $20 \mathrm{~kg}$ ) simulated by a custom-made dynamic loading simulator combining corresponding axial loads with flexion-extension and lateral bending movements. They were applied in oscillating waves at $0.5 \mathrm{~Hz}$ and raised every 100 cycles phase-shifted to each other by $50 \mathrm{~N}$ or $0.25^{\circ}$, respectively. The range of motion (ROM) of the specimens was determined in all three motion planes under pure moments of $3.75 \mathrm{Nm}$ prior to and after implantation as well as subsequently following activities. Simultaneously, subsidence depth was quantified from fluoroscope films. A mixed model (significance level: 0.05) was established to relate subsidence risk to implant geometries and patients' activities.

Results With this new test method, simulating everyday activities provoked clinically relevant subsidence schemes. Generally, severe everyday activities caused deeper subsidence which resulted in increased ROM. Subsidence of lateral end pieces was remarkably less pronounced which was accompanied by a smaller ROM in flexion-extension and higher motion possibilities in axial rotation $(p=0.05)$.

Conclusion In this study, a new biomechanical test method was developed that simulates physiologic activities to examine implant subsidence. It appears that the highest risk of subsidence occurs most when lifting heavy weights, and into the ventral part of the caudal vertebra. The results indicate that lateral end pieces may better prevent from implant subsidence because of the additional cortical support. Generally, patients that are treated with a VBR should avoid activities that create high loading on the spine.
\end{abstract}

Keywords Thoracolumbar spine $\cdot$ Test method $\cdot$ In vitro $\cdot$ Physiological activities $\cdot$ Vertebral body replacements $\cdot$ Sintering

Hans-Joachim Wilke

hans-joachim.wilke@uni-ulm.de

1 Institute of Orthopaedic Research and Biomechanics, Ulm University, Helmholtzstr. 14, 89081 Ulm, Germany

2 Department of Spine Orthopaedics, Orthopaedic University Hospital Friedrichsheim, Frankfurt, Germany

3 Department of Diagnostic and Interventional Radiology, Ulm University Hospital, Ulm, Germany

4 Department of Spine and Reconstructive Orthopaedics, Sana Klinikum Offenbach, Offenbach, Germany

\section{Introduction}

Due to the increase in the prevalence of osteoporosis [1] and the resulting increase in the number of osteoporotic vertebral body fractures, the treatment for these fractures is becoming more important. In addition to conservative therapy, there are less invasive surgical options for cement augmentation, such as vertebroplasty, kyphoplasty and the application of intravertebral force carriers. In case of type A fractures (classified according to Magerl et al. [2]), additive dorsal 
instrumentation or a combination of dorsal instrumentation and vertebral body replacement may be required to restore stability [3, 4]. The poor bone quality of osteoporotic vertebral bodies poses a particular challenge in these surgical procedures. Through further development towards pedicle screws with central cannulation, initial cement augmentation of the screws significantly increased the pull-out force biomechanically and increases the stability between the ventral implant and the vertebral bodies occupied with cement-augmented screws compared to noncement-augmented screw restorations [5, 6]. However, mere augmentation of the pedicle screws does not appear to sufficiently prevent sintering of vertebral replacement implants into the adjacent end plates [3]. Thus, design changes of the vertebral body implants were carried out in order to increase the apophyseal support of the implants on the vertebral endplates.

The present study should now investigate from a biomechanical point of view, whether implants with additional apophyseal support are able to reduce the sintering of vertebral replacement implants during daily life.

In most studies, such vertebral body implants were tested dynamically by applying pure axial loads. Only few studies also represented flexion movements within the dynamic test protocol [7, 8]. For the additional simulation of pure and combined flexion-extension and lateral bending, Kettler and Wilke et al. developed a new test protocol where the axial load ranging from 100 to $600 \mathrm{~N}$ was applied eccentrically at a lever arm of $30 \mathrm{~mm}$ on the specimen mounted on a rotating base platform [9, 10]. However, none of these dynamic loading protocols refers to physiologic loading conditions that occur in vivo during daily life. Therefore, the aim of this study was to develop a new, more physiologic test method which simulates typical daily-life motions of patients being treated with vertebral body implants. With this new test method, typical clinical sintering schemes should be replicated and related to patients' activities. This should further allow for evidence-based recommendations for post-operative therapy and rehabilitation of patients with vertebral body replacements.

\section{Methods}

\section{Specimens}

Twelve fresh frozen human thoracolumbar spinal segments (T11-L1, L2-L4) were obtained from ten different donors (median age 78 years (36-83 years), median bone mineral density (BMD) (64.2 mgCaHA/ $\mathrm{cm}^{3}$ (0.0-103.6 mgCaHA/ $\mathrm{cm}^{3}$ ) 6f:4 m). Quantitative computed tomography (QCT, Siemens Somatom Definition AS +, Siemens Healthineers Erlangen, Germany) was performed to measure the trabecular BMD. Computed tomography (CT, Siemens Somatom Definition AS +, Siemens Healthineers Erlangen, Germany) scans were taken to identify possible fractures, tumours and other disorders that might influence the biomechanical properties. All remaining spines have been included and prepared for testing. All soft tissue was removed preserving all bony structures, the intervertebral discs, ligaments and facet capsules. Screws were inserted into the cranial endplate of the upper and the caudal endplate of the lower vertebrae before embedding in polymethylmethacrylate (PMMA, Technovit 3040, Heraeus Kulzer, Wehrheim, Germany). Flanges were mounted on the PMMA embedment for proper mounting into the testing machines. The specimens were then stratified into two groups by median age ((a) 78 years (36-83 years), (b) 73.5 years (36- 87 years)) and median BMD ((a) $64.2 \mathrm{mgCaHA} / \mathrm{cm}^{3}$ (49.3-92.2 mgCaHA/ $\left.\mathrm{cm}^{3}\right)$, (b) $\left.66.7 \mathrm{mgCaHA} / \mathrm{cm}^{3}\left(0.0-103.6 \mathrm{mgCaHA} / \mathrm{cm}^{3}\right)\right)$, as well as segment level (3:3) and sex (3:3) (Fig. 1). Until testing, specimens were stored in triple sealed polyethylene bags at $-20{ }^{\circ} \mathrm{C}$. Prior to testing, the specimens have been carefully thawed overnight at $+4{ }^{\circ} \mathrm{C}$.
Fig. 1 Distribution of BMD and age of the specimens in the two groups: implantation of vertebral body replacements with round, centrally located endplates, BMD 64.2 mgCaHA/ $\mathrm{cm}^{3}$ (49.3-92.2), age 78 yrs (36-83) (blue) or lateral endplates with apophyseal support, BMD 66.7 mgCaHA/ $\mathrm{cm}^{3}(0.0-103.6)$, age $73.5 \mathrm{yrs}$ (36-87) (yellow)

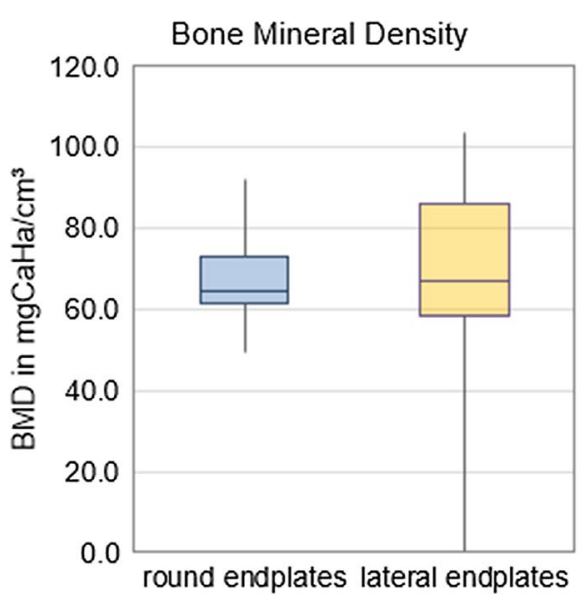




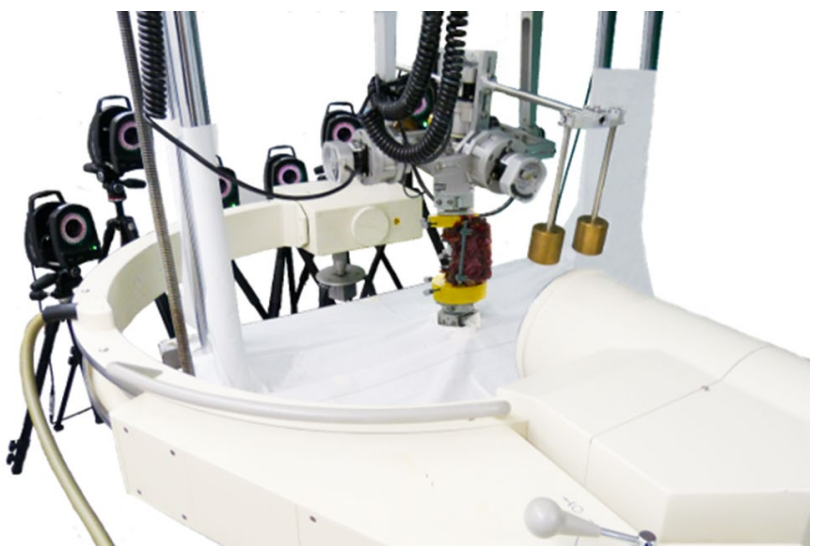

Fig. 2 Universal spine tester [11] where the flexibility test was performed to measure ROM by applying pure moments of $\pm 3.75 \mathrm{Nm}$ in flexion-extension, lateral bending and axial rotation

\section{Test protocol}

Range of motion (ROM) and neutral zone (NZ) were measured during a quasistatic flexibility test in a universal spine tester [11] (Fig. 2) at three phases of the experiment: first in the intact state, then postoperatively and finally after dynamic loading simulating different physiologic activities. Pure moments of $\pm 3.75 \mathrm{Nm}$ were applied in flexion-extension, lateral bending and axial rotation. ROM and NZ were assessed by simultaneous optical motion tracking with the Vicon MX13 system (Vicon Motion Systems Ltd., Oxford, UK). While performing the flexibility tests, fluoroscope films (Fig. 2) were taken to assess subsidence depth of the implants inside the adjacent vertebrae. The subsidence depth was measured in flexion, extension, left and right lateral bending, for the implant subsidence into the anterior and posterior part of the caudal and cranial adjacent vertebrae.

After assessing the intact biomechanical properties of the specimens, a partial corpectomy and implantation of the vertebral body replacement were performed. A posterior rod-screw stabilisation system was implanted into the cranial and caudal vertebra of the specimen with cement augmentation of the pedicle screws with $2 \mathrm{ml}$ cement per screw. Subsequently, approximately two-thirds of the midvertebra (T12 or L3, respectively) and the adjacent discs were removed keeping the last third of the posterior cortical wall intact. The expandable vertebral body replacement was implanted and distracted until the original height of the mid vertebral body and discs was reached. According to the group affiliation of the specimens (Fig. 1), the vertebral body implants for each specimen were either equipped with (1) round, centrally located endplates or (2) so-called lateral endplates that have a rectangular shape and thus additional apophyseal support. Generally, for the replacement of the thoracic vertebrae (T12), round endplates with the diameter of $29 \mathrm{~mm}$ and lateral endplates with a width of $45 \mathrm{~mm}$ were used. For the replacement of the lumbar vertebrae (L3), a diameter of $32 \mathrm{~mm}$ and width of $55 \mathrm{~mm}$ were used, respectively. The angulation of the endplates was chosen individually according to the lordosis of each specimen.

For the simulation of the different physiologic activities, a new test method was developed using the dynamic spine loading machine [12] in order to combine motion and axial loading that acts on the spine during those activities.

To determine the axial load that usually acts on a healthy spine during daily-life activities, in vivo intradiscal pressure measurements from Wilke et al. were used and converted into axial loading with conversion factors proposed by Brinckmann and Grootenboer (Fig. 3, [14]). These conversion factors depend on the anatomical differences of the transverse cross section of the individual intervertebral disc of the patient and therefore varied between 1.3 and 1.8 [14]. For the simulation of climbing stairs and tying shoes, the minimum calculated loads were used. The worst-case scenario of lifting heavy weights (e.g. a box of $20 \mathrm{~kg}$ ) was simulated by applying the maximum load. In each protocol, the load increased in steps of $100 \mathrm{~N}$ each 100 cycles from the maximum load of the latter tested activity to the maximum load of the following tested activity, starting with $500 \mathrm{~N}$ for a relaxed standing patient (Figs. 3 , 4). Superimposed on the axial load was rotation in flexion-extension and left-right lateral bending, which was applied at phase-shifted sine and cosine waves, thus producing a complex coupled threedimensional movement. The rotational motion increased in steps of $0.25^{\circ}$ each 100 cycles until a maximum value of $1^{\circ}$, phase-shifted to the load increase. The load and motion cycles were applied at a frequency of $0.5 \mathrm{~Hz}$.

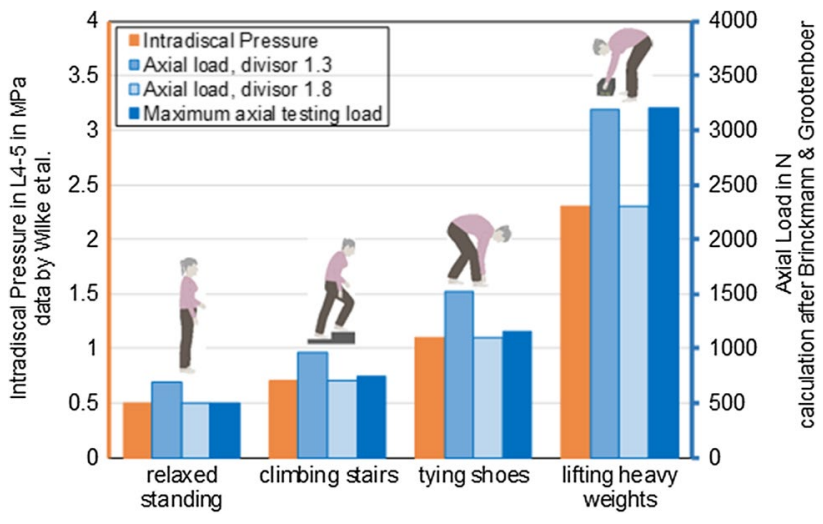

Fig. 3 Calculated axial loads in $\mathrm{N}$ for the simulation of the physiological activities (relaxed standing, climbing stairs, tying shoes and lifting heavy weights), based on intradiscal pressure data acquired by Wilke et al. [13] and converted with factors proposed by Brinckmann and Grootenboer, 1991 [14] 

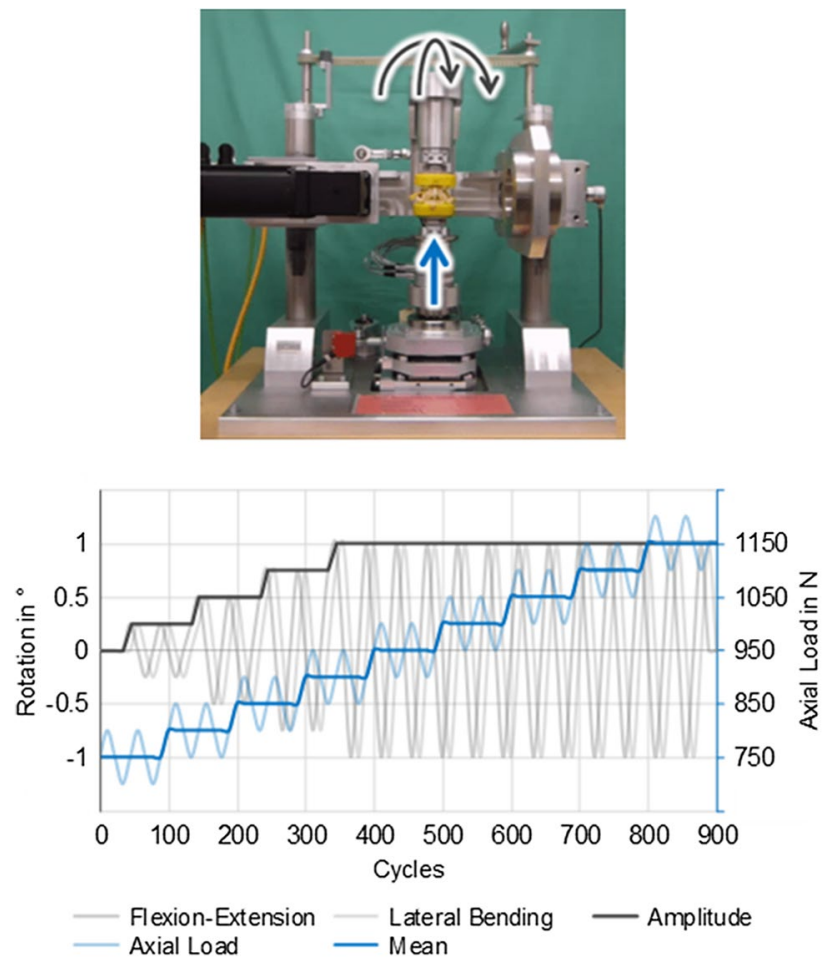

Fig. 4 Dynamic spine loading simulator and sinusoidal loading scheme used for the simulation of the physiological activities, exemplarily shown for tying shoes starting with axial loading of the previous activity only (750 $\mathrm{N}$ for climbing stairs) and rising the rotation angle and the axial load phase-shifted to each other until the maximum values are reached $\left(1150 \mathrm{~N}\right.$ axial load for tying shoes and $\pm 1^{\circ}$ rotation)

\section{Statistical analysis}

Statistical analysis was performed with SPSS (IBM® SPSS ${ }^{\circledR}$ Statistics Version 24; IBM Corp., Armonk, NY) for the ROM and NZ measurements, as well as subsidence depth of the tested segments in every testing condition. Normal distribution could not be proven by Shapiro-Wilk test with a $p$ value of 0.05 . Therefore, median and ranges are provided for all data and nonparametric tests for distribution-free data were performed. Significant differences and dependencies from BMD, age and segment were assessed using a linear mixed model with a significance level set to $\alpha=0.05$.

\section{Results}

A new test method for the simulation of everyday activities such as climbing stairs, tying shoes and lifting heavy weights (up to $20 \mathrm{~kg}$ ) could be developed and validated. In the dynamic loading protocol of this test method, axial loads that were calculated from in vivo intradiscal pressure measurements were applied to the fused specimens. Additionally, combined motions of up to $\pm 1^{\circ}$ in flexion-extension and lateral bending were created in order to provoke circumferential subsidence effects. This test method was successfully used to provoke typical subsidence schemes that are reported clinically (Fig. 5). Macroscopically, signs of subsidence could be seen in both groups with the different endplate geometries. Implants with round endplates subsided remarkably deeper into the adjacent vertebra than their lateral endplate implant counterparts, which primarily began
Fig. 5 Exemplary subsidence behaviour of round and lateral endplates in the lateral view

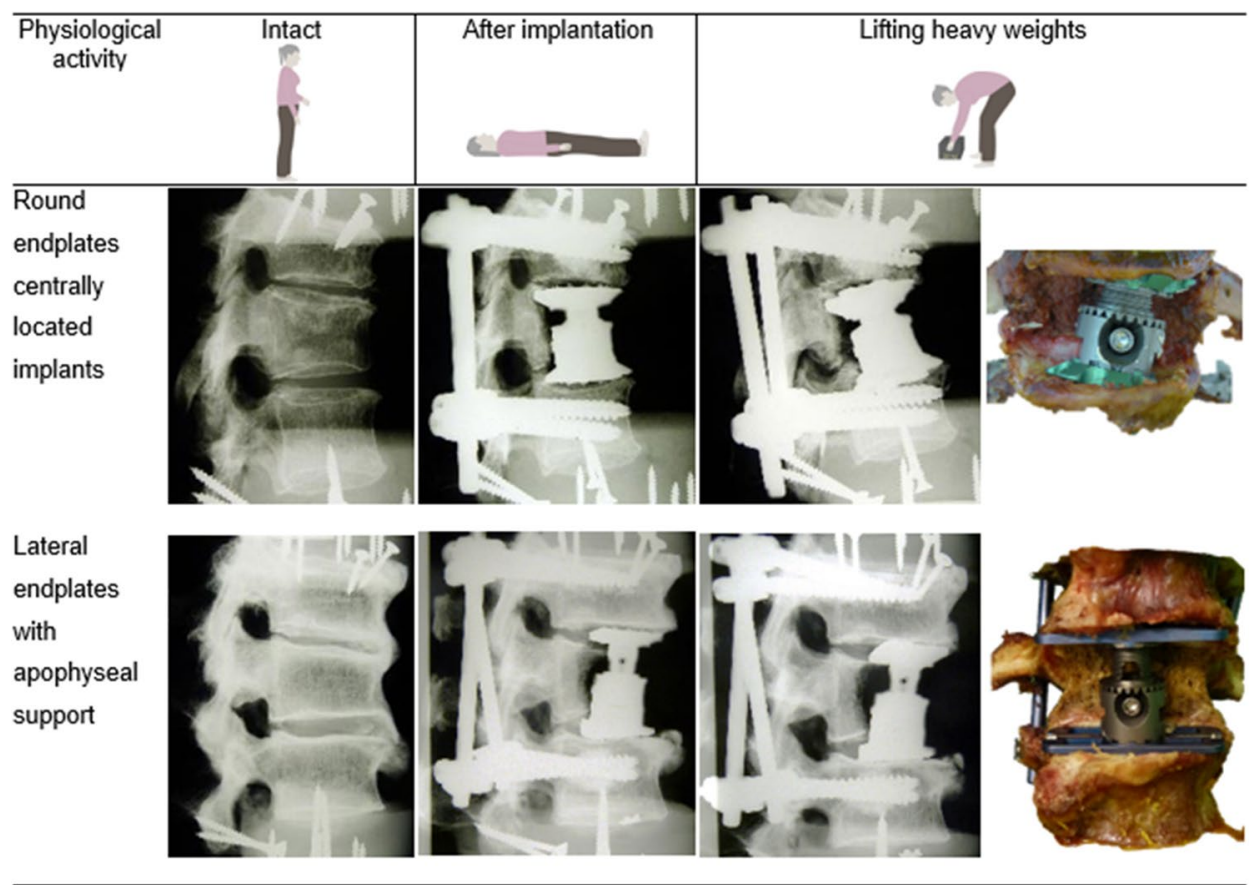


subsiding when simulating lifting heavy weights. This difference presumably derives from the additional apophyseal support gained by the geometry of the endplate. The subsidence depth only showed noticeable tendencies for implant subsidence in flexion and only into the caudal adjacent vertebra (Fig. 6). The initial subsidence depth was evaluated after implantation. The implants with round endplates have been implanted into the segments with proper posterior fixation of $0.0 \mathrm{~mm}$ subsidence into the vertebrae, whereas the anterior part was implanted slightly loose without any contact to the vertebral endplate $(1.5 \mathrm{~mm})$. In the segments with lateral endplate geometry, the anterior part was slightly loose, as well $(0.7 \mathrm{~mm})$ and the posterior part proper fitted $(0.0 \mathrm{~mm})$. Subsidence increased further with more severe loading conditions during the physiological activities. The simulation of lifting heavy weights finally led to a subsidence of $-4.2 \mathrm{~mm}$ in the anterior part of the caudal vertebra and $-3.8 \mathrm{~mm}$ into the posterior part for the round endplates, compared to the initial position of the implant. Interestingly, the lateral endplates subsided $-0.7 \mathrm{~mm}$ and $-1.8 \mathrm{~mm}$, into the anterior and posterior part of the caudal vertebra, respectively. An increased subsidence predominantly into the caudal vertebra was first observed when simulating stair climbing of specimen installed with round endplates, where this effect was not seen in the lateral endplate group. Subsidence progressed into the posterior aspect of the caudal vertebra, generating a more pronounced imprint after simulating tying shoes.

Vertebral body replacement implantation was successful in all specimens achieving primary stability with only $\pm 1^{\circ}$ remaining ROM in all motion planes in both implant shape groups (Fig. 7). The simulation of daily-life activities reversed initial stabilisation, resulting in decreased fusion and increased ROM. The increase was more pronounced with more severe loading conditions of the different activities. The simulation of climbing stairs led to a steady increase of ROM in all motion planes for both endplate geometries to median values from $\pm 1.3^{\circ}$ to $\pm 1.9^{\circ}$. After simulating tying shoes, flexion-extension and lateral bending ROM increased in both groups, again, with median values ranging from $\pm 3.3^{\circ}$ to $\pm 4.3^{\circ}$. In axial rotation, the stepwise increase in ROM across simulations was smaller for both groups with a larger observed for the lateral endplates $\left(2.3^{\circ}\right.$ for left axial rotation and $-2.4^{\circ}$ for right axial rotation), versus $\pm 1.9^{\circ}$ in the round endplates.

Axial rotation, ROM further increased after simulating heavy weightlifting, where left axial rotation increased to $3.1^{\circ}$ versus $1.7^{\circ}(p=0.108)$ for the lateral endplates and round endplates, respectively. Right axial rotation ROM increased to $-3.3^{\circ}$ versus $-1.7^{\circ}(p=0.05)$.

In general, no differences of the results of the ROM nor subsidence depth showed statistically dependence on BMD, instrumented level or age of the specimens.

\section{Discussion}

In the present study, a new in vitro test method was developed that permits dynamic simulation of distinct physiologic loading scenarios of patients' daily life. In comparison with previous dynamic test methods, this new test method replicates typical activities that a patient could perform after fusion and vertebral body replacement. Previous studies usually examined fused segments or vertebral body implants by applying either pure axial loads $[15,16]$ or applying axial loads in the anterior part of the specimen [7, 8]. In the latter case also, flexion movements were simulated dynamically.
Fig. 6 Subsidence depth in $\mathrm{mm}$ into the a posterior and $\mathbf{b}$ anterior part of the caudal adjacent vertebra of round and lateral endplates after implantation and after the simulation of climbing stairs, tying shoes and lifting heavy weights, assessed with fluoroscope films taken while the flexibility test in flexionextension
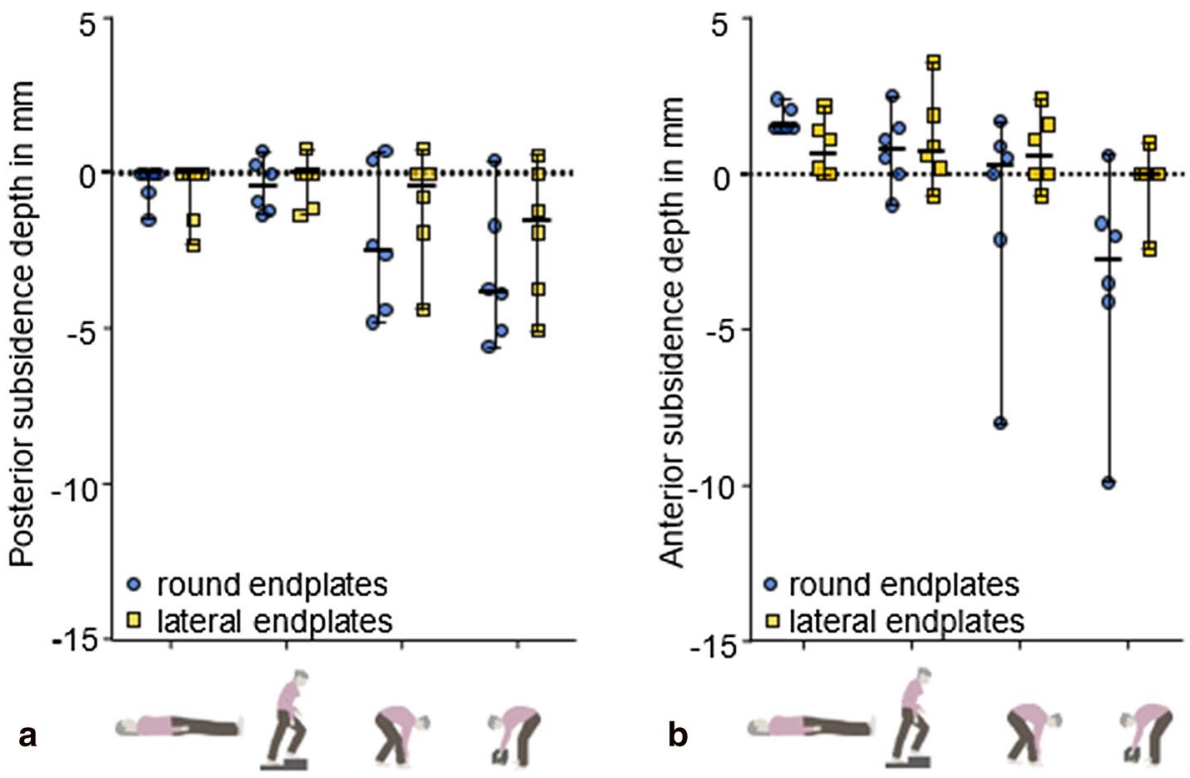
Fig. 7 Range of motion (ROM) and neutral zone (NZ) of round and lateral endplates in $\mathbf{a}$ flexion-extension, b left/right lateral bending and $\mathbf{c}$ in left/ right axial rotation in the intact state, after implantation and after the simulation of climbing stairs, tying shoes and lifting heavy weights, assessed with the universal spine tester applying pure moments of $\pm 3.75 \mathrm{Nm}$, respectively. ${ }^{*} p$-value $\leq 0.05$
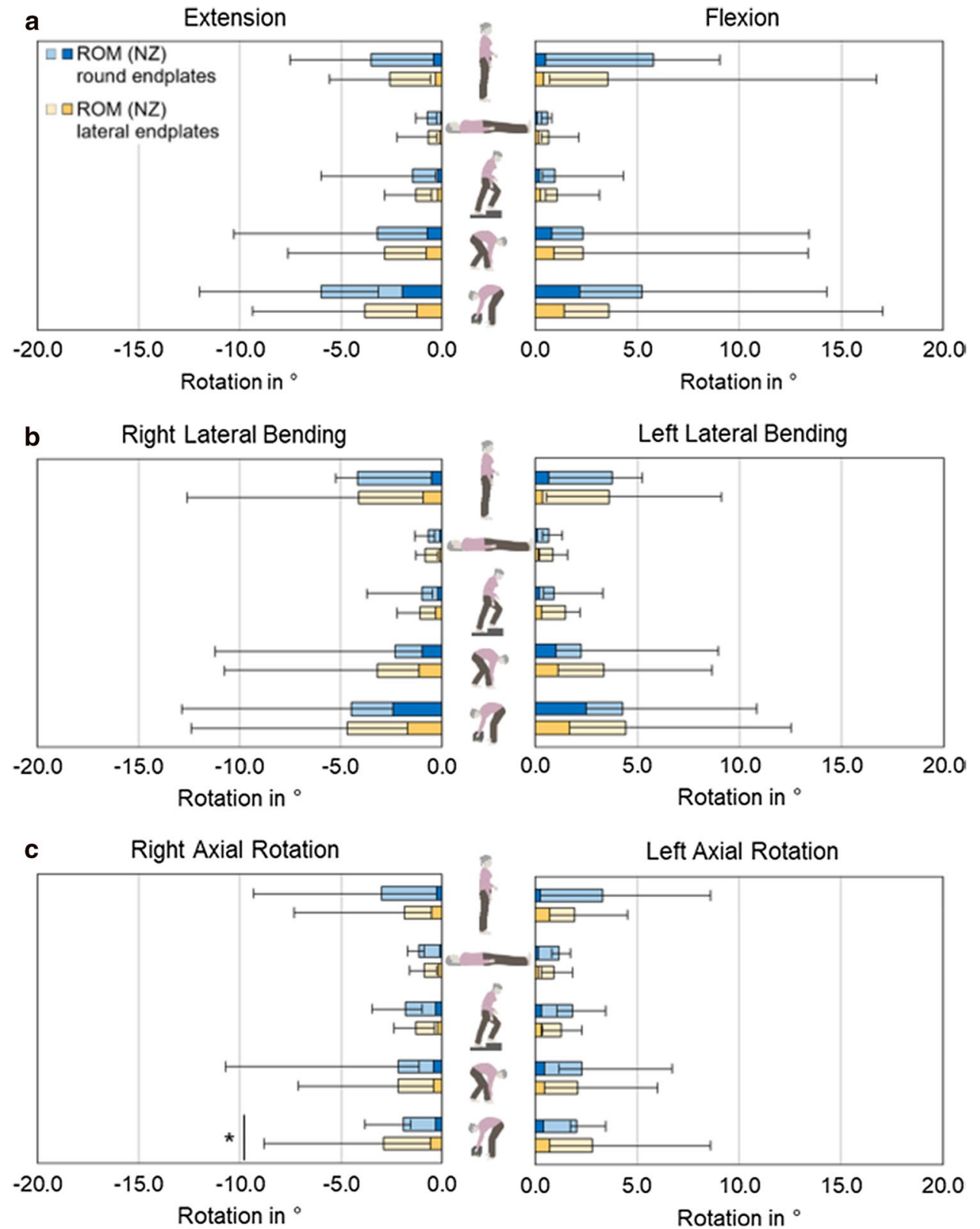

However, this did not completely represent the complexity of spinal loading scenarios that occur during daily-life activities. With the new dynamic test method, such activities could be simulated directly. Influences that result from combined flexion-extension and lateral bending could be examined as well. Motion in axial rotation was not be applied in this study due to the very small ROM compared to the ROM in other motion directions in the (thoraco)lumbar spine [17-19]. Furthermore, the loads that were applied could be directly related to the simulated activities. However, these activities are only symbolic and may vary greatly under realistic conditions. The activities that were replicated in this study addressed the clinical issue of implant subsidence after treatments with vertebral body replacements. For other studies, relevant postures and activities should be carefully selected according to the clinical setting. Additionally, in vivo data about forces that act on a spine during motion cannot be easily achieved. The forces highly depend on the anatomy as well as the individual spine [14]. This was taken into account by applying certain ranges of the axial load that was calculated from in vivo intradiscal pressure values [13] using different conversion factors [14].

Because of our ageing society, osteoporosis and hence the treatment for osteoporotic spinal fractures are highly clinically relevant. With the new test method, it was possible to provoke clinical subsidence patterns of vertebral endplates into osteoporotic spinal segments and further, to predict the risk of implant subsidence depending on different 
physiological activities. Based on the results gained with this study, an optimisation of the treatment for each individual patient could be reached by careful selection of the vertebral body replacement regarding geometry, size and angulation as well as proper implantation. In this context, the subsidence risk of different endplate geometries of the implants has been investigated. It seemed that the risk of implant subsidence is lower, the higher the apophyseal support. During this study, severe complications like very deep subsidence or implant dislocation [20-24] that are reported in clinics and would require reoperation could not be observed in the segments treated with the lateral endplates, but with round endplates. With increased loads while simulating physiological activities, rather a proper fixation than subsidence could be seen between in the lateral implant towards the vertebral endplate. Particularly when taking into account the high initial instability of the lateral endplate group, these segments were sufficiently stabilised by the implantation, as well. The instability that resurfaced during the simulation of the daily-life activities seemed to happen predominantly when performing activities with high movements in flexion such as tying shoes or lifting heavy weights. On the contrary, in the round endplate group, instability because of subsidence began to resurface already when climbing stairs. Interestingly, the severe implant subsidence of the round endplate group further resulted in a blocking in axial rotation. Less implant subsidence like what could be observed in vertebral body replacements with lateral endplates still allowed a certain range of motion in axial rotation.

Regarding the development of the new test method, limitations result from the conversion of the intradiscal pressure into the axial loads. The divisor for calculating the axial loads acting on the segments was not adjusted for every segment. Brinckmann and Grootenboer proposed different conversion factors for individual segments [14] but the conversion depends on the cross section of each individual disc. In this study, 12 individual segments from ten donors were used which generated a high anatomical variability. This was one reason why the whole range of conversion factors were covered by setting up a standardised loading protocol with increasing loads. Additionally, that the true axial forces that act on an intact segment are not the same as the axial forces that act on the implant. Because of the smaller cross section of the implant, the actual axial force acting on the implant may be higher.

Limitations raised from the heterogeneous sample that has been tested. However, the specimens were divided into the two groups, stratified equally by important biomechanical characteristics including BMD, age, segment and sex. Furthermore, the group with the wider range of BMD values and the worst specimen (trabecular BMD of 0 mgCaHA/ $\mathrm{cm}^{3}$ ) was set to be treated with the lateral endplate. This endplate geometry was hypothesised to have the better surgical outcome because of the additional apophyseal support. Besides this, the implantation was performed and the instrumentation was then visually checked by the same and experienced spine surgeon in order to minimise deviations in implant positioning. Furthermore, the instrumentation of the specimens was performed blindly until the point of insertion of the vertebral body replacement in order to minimise a bias during the implantation procedure.

Another limitation lies in possible screw loosening within the osteoporotic bone which may have been provoked by the aggressive test method producing deeper subsidence. However, screw loosening is a known complication in osteoporotic patients $[25,26]$ and therefore should not depend on the vertebral replacement geometry.

\section{Conclusion}

In this study, a new physiological test method was developed and used to provoke relevant subsidence patterns of vertebral body replacements that are reported in the clinical setting. In this context, it seems that vertebral body implants with lateral endplates provide superior apophyseal support compared to round, centrally located endplates. Furthermore, this study shows that avoiding extreme flexion and heavy weight lifting should be avoided during post-operative physical therapy in order to maintain implant fixation.

Funding Open Access funding enabled and organized by Projekt DEAL. This study was funded by the German Spine Foundation and partially by the German Research Foundation (WI 1352/14-3).

\section{Compliance with ethical standards}

Conflicts of interest The vertebral body replacement implants and posterior stabilisation systems were provided by ulrich $\mathrm{GmbH} \& \mathrm{Co} . \mathrm{KG}$, Ulm, Germany.

Ethics approval The study was approved by the ethical committee board of the University of Ulm (No. 142/17).

Availability of data and material Informed.

Open Access This article is licensed under a Creative Commons Attribution 4.0 International License, which permits use, sharing, adaptation, distribution and reproduction in any medium or format, as long as you give appropriate credit to the original author(s) and the source, provide a link to the Creative Commons licence, and indicate if changes were made. The images or other third party material in this article are included in the article's Creative Commons licence, unless indicated otherwise in a credit line to the material. If material is not included in the article's Creative Commons licence and your intended use is not permitted by statutory regulation or exceeds the permitted use, you will need to obtain permission directly from the copyright holder. To view a copy of this licence, visit http://creativecommons.org/licenses/by/4.0/. 


\section{References}

1. Forstein DA, Bernardini C, Cole RE, Harris ST, Singer A (2013) Before the breaking point: reducing the risk of osteoporotic fracture. J Am Osteopath Assoc 113(2 Suppl 1):S5-24

2. Magerl F, Aebi M, Gertzbein SD, Harms J, Nazarian S (1994) A comprehensive classification of thoracic and lumbar injuries. Eur Spine J 3(4):184-201

3. Geiger F, Kafchitsas K, Rauschmann M (2011) Anterior vertebroplasty of adjacent levels after vertebral body replacement. Eur Spine J 20(8):1385-1392

4. Spiegl U, Jarvers JS, Heyde CE, Josten C (2017) Osteoporotic vertebral body fractures of the thoracolumbar spine: indications and techniques of a 360 degrees - stabilization. Eur J Trauma Emerg Surg 43(1):27-33

5. Burval DJ, McLain RF, Milks R, Inceoglu S (2007) Primary pedicle screw augmentation in osteoporotic lumbar vertebrae: biomechanical analysis of pedicle fixation strength. Spine (Phila Pa 1976) 32(10):1077-1083

6. Tan JS, Bailey CS, Dvorak MF, Fisher CG, Cripton PA, Oxland TR (2007) Cement augmentation of vertebral screws enhances the interface strength between interbody device and vertebral body. Spine (Phila Pa 1976) 32(3):334-341

7. Disch AC, Knop C, Schaser KD, Blauth M, Schmoelz W (2008) Angular stable anterior plating following thoracolumbar corpectomy reveals superior segmental stability compared to conventional polyaxial plate fixation. Spine (Phila Pa 1976) 33(13):1429-1437

8. Oberkircher L, Krüger A, Hörth D, Hack J, Ruchholtz S, Fleege C, Rauschmann M, Arabmotlagh M (2018) Anterior cement augmentation of adjacent levels after vertebral body replacement leads to superior stability of the corpectomy cage under cyclic loading-a biomechanical investigation. Spine J 18(3):525-531

9. Kettler A, Schmölz W, Shezifi Y, Ohana N, Ben-Arye A, Claes L, Wilke HJ (2006) Biomechanical performance of the new BeadEx implant in the treatment of osteoporotic vertebral body compression fractures: Restoration and maintenance of height and stability. Clin Biomech 21(7):676-682

10. Wilke HJ, Mehnert U, Claes LE, Bierschneider MM, Jaksche H, Boszczyk BM (2006) Biomechanical evaluation of vertebroplasty and kyphoplasty with polymethyl methacrylate or calcium phosphate cement under cyclic loading. Spine 31(25):2934-2941

11. Wilke HJ, Claes L, Schmitt H, Wolf S (1994) A universal spine tester for in vitro experiments with muscle force simulation. Eur Spine J 3(2):91-97

12. Wilke HJ, Kienle A, Maile S, Rasche V, Berger-Roscher N (2016) A new dynamic six degrees of freedom disc-loading simulator allows to provoke disc damage and herniation. Eur Spine J 25(5):1363-1372

13. Wilke HJ, Neef P, Caimi M, Hoogland T, Claes LE (1999) New in vivo measurements of pressures in the intervertebral disc in daily life. Spine 24(8):755-762
14. Brinckmann P, Grootenboer H (1991) Change of disc height, radial disc bulge, and intradiscal pressure from discectomy. An in vitro investigation on human lumbar discs. Spine (Phila Pa 1976) 16(6):641-646

15. Kettler A, Wilke HJ, Dietl R, Krammer M, Lumenta C, Claes L (2000) Stabilizing effect of posterior lumbar interbody fusion cages before and after cyclic loading. J Neurosurg 92(1 Suppl):87-92

16. Knop C, Lange U, Bastian L, Oeser M, Blauth M (2001) Biomechanical compression tests with a new implant for thoracolumbar vertebral body replacement. Eur Spine J 10(1):30-37

17. Panjabi MM, White AA 3rd (1980) Basic biomechanics of the spine. Neurosurgery 7(1):76-93

18. Wilke HJ, Herkommer A, Werner K, Liebsch C (2017) In vitro analysis of the segmental flexibility of the thoracic spine. PLoS ONE 12(5):e0177823

19. Wilke HJ, Wolf S, Claes LE, Arand M, Wiesend A (1995) Stability increase of the lumbar spine with different muscle groups A biomechanical in vitro study. Spine (Phila Pa 1976) 20(2):192-198

20. Arts MP, Peul WC (2008) Vertebral body replacement systems with expandable cages in the treatment of various spinal pathologies: a prospectively followed case series of 60 patients. Neurosurgery 63(3):537-544, discussion 544-535

21. Lange U, Edeling S, Knop C, Bastian L, Oeser M, Krettek C, Blauth M (2007) Anterior vertebral body replacement with a titanium implant of adjustable height: a prospective clinical study. Eur Spine J 16(2):161-172

22. Lange U, Knop C, Bastian L, Blauth M (2003) Prospective multicenter study with a new implant for thoracolumbar vertebral body replacement. Arch Orthop Trauma Surg 123(5):203-208

23. Mohammad-Shahi MH, Nikolaou VS, Giannitsios D, Ouellet J, Jarzem PF (2013) The effect of angular mismatch between vertebral endplate and vertebral body replacement endplate on implant subsidence. J Spinal Disord Tech 26(5):268-273

24. Uchida K, Kobayashi S, Nakajima H, Kokubo Y, Yayama T, Sato R, Timbihurira G, Baba H (2006) Anterior expandable strut cage replacement for osteoporotic thoracolumbar vertebral collapse. J Neurosurg Spine 4(6):454-462

25. Ponnusamy KE, Iyer S, Gupta G, Khanna AJ (2011) Instrumentation of the osteoporotic spine: biomechanical and clinical considerations. Spine J 11(1):54-63

26. Wu ZX, Gong FT, Liu L, Ma ZS, Zhang Y, Zhao X, Yang M, Lei W, Sang HX (2012) A comparative study on screw loosening in osteoporotic lumbar spine fusion between expandable and conventional pedicle screws. Arch Orthop Trauma Surg 132(4):471-476

Publisher's Note Springer Nature remains neutral with regard to jurisdictional claims in published maps and institutional affiliations. 